\title{
Rho GTPases at the crossroad of signaling networks in mammals
}

\author{
Jean Claude Hervé ${ }^{1, *}$ and Nicolas Bourmeyster,"*
}

'INSERM U1082; Université de Poitiers; Poitiers Cédex, France; ${ }^{2}$ Laboratoire Signalisation et Transports loniques Membranaires (STIM); Université de Poitiers Poitiers Cédex, France

The following collection of reviews concerning Rho GTPases was elaborated in the perspective of a special anniversary. 30 years ago, Pascal Madaule, postdoc in Richard Axel's laboratory at Columbia University, was searching for homologs of mammalian hormones in Aplysia. One of the clones presented a strong sequence homology to Ras, and was then termed Rho for Ras Homologous. ${ }^{1}$ Then they established that this new protein was evolutionary conserved from yeast to Humans. This discovery opened several new fields of research. Firstly, back in France, Pascal Madaule and his students discovered many other small GTPases by sequence homology and new protein families were built (Ras, Rho, Rab, ARF, ...). More than one hundred members of small GTPase family are now known. Secondly, very rapidly after their discovery, Rho family GTPases major functions were determined thanks to the bacterial toxins developed in parallel ${ }^{2}$. Pierre Chardin, an ex-student of Pascal Madaule, in collaboration with Patrice Boquet and Michel Popoff enlightened the role of Rho in actin cytoskeleton organization. ${ }^{3}$ After these fundamental discoveries, the next milestone was to determine the specific roles of RhoA, Rac1 and Cdc42, the three canonical members of Rho family GTPases, in very specific reorganizations of actin cytoskeleton (i.e. stress fibers, lamellipodia and filopodia). ${ }^{4,5}$ During the last 30 years, the landscape has been enlarged and depixelated, Rho GTPases are now established key regulators of many fundamental cellular processes, involved in each cellular process which depends on cytoskeletal organization (e.g. the regulation of actin cytoskeleton, cell division, and cell motility), but also in gene expression, vesicle trafficking and endocytosis. The Rho family consists of 22 genes in humans, encoding at least 25 signaling proteins.

It was time to try to gather most of the accumulated knowledge about these central actors of so many different signaling pathways. This special issue of Small GTPases is divided into six focus article sections depicting Rho family GTPases from biochemistry to physiopathology.

\section{Factors Influencing the Activities of the Rho Family of GTPases}

Rho GTPases are activated by Rho Guanine nucleotide Exchange Factors (GEF), which catalyze the release of GDP thus allowing GTP to bind. In mammals, there are two distinct GEF families for Rho proteins: those of the diffuse B-cell lymphoma (Dbl) and dedicator of cytokinesis (Dock) families. Susanne Schmidt and Anne Debant ${ }^{6}$ focus on the function and regulation of the Dbl family RhoGEF Trio, a complex protein with multiple domains. The Trio protein and its paralog Kalirin are unique within this family in that they harbor two GEF domains of distinct specificity. Their structural organization is very well conserved through evolution. Trio plays important roles in cell adhesion and in signaling pathways elicited by $\mathrm{G} \alpha \mathrm{q}$ protein-coupled receptors. Trio appears as a major actor in cellular physiology, a regulator of neuronal development. Its expression is significantly increased in different types of tumors, suggesting that it might be involved in oncogenesis. Vav proteins (Vav1, Vav2 and
Vav3) are members of the Dbl family of GEFs for Rho GTPases, hierarchically located downstream of protein tyrosine kinases. In addition, they also exhibit various catalysis-independent roles in specific signaling contexts. They play essential signaling roles for the development and/or effector functions of a large variety of cell lineages, including those belonging to the immune, nervous, and cardiovascular systems. They also contribute to pathological states such as cancer, immune-related dysfunctions, and atherosclerosis. Xosé R Bustelo $^{7}$ provides an integrated view about the evolution, regulation, and effector properties of these signaling molecules and about their potential consideration as therapeutic targets. P-Rex $\left(\mathrm{PI}(3,4,5) \mathrm{P}_{3}\right.$ dependent Rac exchanger) proteins are other Rac-specific members of the Dbl family. Rac GTPases regulate a wide range of cell functions, including cell shape, movement, secretion, phagocytosis, transcription, translation, and the production of reactive oxygen species (ROS). P-Rex proteins derive from two genes, PREX1 (encoding P-Rex1) and PREX2 (which encodes two proteins, full-length P-Rex2 (also known as P-Rex2a) and the splice variant P-Rex $2 b$ which lacks the $\mathrm{C}-1$ terminal half). Data from genetically- modified mice revealed that P-Rex1 has physiological importance in the inflammatory response and the migration of melanoblasts during development whereas PRex 2 controls the dendrite morphology of cerebellar Purkinje neurons as well as glucose homeostasis in liver and adipose tissues. Heidi C.E. Welch ${ }^{8}$ examines the regulation and function of P-Rex proteins.

Oxidation and reduction events contribute to a plethora of physiological and

This issue is dedicated to the memory of Pr Alan Hall who died on May 3rd 2015

*Correspondence to: Jean Claude Hervé; Email: jean.claude.herve@univ-poitiers.fr; Nicolas Bourmeyster; Email: n.bourmeyster@chu-poitiers.fr http://dx.doi.org/10.1080/21541248.2015.1044811 
patho-physiological processes because they modulate, in part, activation or inactivation of various redox sensitive proteins. At moderate concentrations, reactive nitrogen and oxygen species play key roles as regulatory mediators in signaling processes. Cysteine residues in proteins are among their well known targets, and cysteine oxidation appears, as posttranslational modification of Rho GTPases, to drive differences in their localization and activity. On the other hand, Rho GTPases regulate the cellular redox state by controlling enzymes that generate and convert reactive oxygen and nitrogen species. G.A. Hobbs, B. Zhou, A.D. Cox and S.L. Campbell $^{9}$ explain how redox agents may act both as upstream regulators and as downstream effectors of Rho GTPases.

The Bin/Amphiphysin/Rvs (BAR) domain proteins form a superfamily of proteins implicated in fundamental biological processes by sensing and inducing membrane curvature. Through their capacity to bind lipids as well as proteins, BAR domains are involved in the targeting of proteins to specific regions within the plasma membrane where actin remodeling is necessary. At these sites, BAR domain proteins can modulate Rho GTPase activity, either by regulating the activation status of Rho GTPases since some BAR domain proteins also harbor a RhoGAP/ GEF domain, or by linking Rho GTPases to their activators or to their effectors. Pontus Aspenström ${ }^{10}$ explains how Rho GTPases are key factors for orchestration of this intricate interplay.

Given the numerous mechanisms that regulate the activity of Rho GTPases and the multiple effectors for Rho proteins, how is specificity achieved when transducing signals via Rho GTPase-regulated molecular networks? Rho proteins comprise the core G domain (or "switch region", involved in GTP binding and hydrolysis), a short 'insert region' and the C-terminal short hypervariable region. The available evidence supports the notion that multiple regions in RhoA/B/C cooperate to provide selective interactions with upstream regulators and downstream effectors, that these interactions may involve several portions of the GTPase at the same time. These specific interactions are highly regulated in time and space.
Antje Schaefer, Nathalie R. Reinhard and Peter L. Hordijk ${ }^{11}$ discuss the key features that contribute to this RhoGTPase specificity.

\section{Major Effector Proteins for the Rho GTPases}

Rho-associated coiled-coil containing kinases (ROCK) are kinases belonging to the AGC (PKA/ PKG/PKC) family of serine-threonine kinases. They were the first discovered Rho effectors, involved in RhoA-mediated stress fiber formation and focal adhesion assembly by enhancing phosphorylation of myosin light chain. It later appeared that the ROCK kinases actively phosphorylate a large cohort of actin-binding proteins and intermediate filament proteins to modulate their functions. Two mammalian ROCK homologs have been identified, ROCK1 (also called ROCK I, ROK $\beta$, Rho-kinase $\beta$, or p160ROCK) and ROCK2 (also known as ROCK II, ROK $\alpha$, or Rho kinase), with however an extensive homology between their two catalytic domains. Linda Julian and Michael F. Olson ${ }^{12}$ overview the structure, regulation, and functions of ROCKs. As ROCKs, myotonic dystrophy kinase-related Cdc42-binding kinases (MRCKs) constitute a protein kinase subfamily belonging to the AGC kinase superfamily. The MRCK subfamily is a downstream effector of another Rho family GTPase, Cdc42, the key regulator of cell polarity and directional migration. It consists of three members, MRCK $\alpha$, MRCK $\beta$ and MRCK $\gamma$. Despite sharing several common substrates, particularly myosin light chain (MLC), whose ROCKs and MRCKs promote phosphorylation either directly or indirectly by inhibiting the MLC phosphatase, ROCKs and MRCKs are independently regulated and undertake different functions. ZhuoShen Zhao and Ed Manser ${ }^{13}$ summarize the knowledge about MRCKs and highlight the similarities and differences between MRCK and ROCK kinases.

The assembly of actin monomers into filamentous structures does not occur spontaneously but requires factors which help to overcome the kinetic barrier of nucleation, the formation of a stable actin polymer from its monomeric units. Formins are a group of 15 proteins in mammals capable of actin nucleation and able to promote the elongation of pre-existing filaments by removing barbed end capping proteins. A subgroup of mammalian formins, termed Diaphanous-related formins or DRFs, are autoinhibited in the resting state by an $\mathrm{N}$ - to $\mathrm{C}$-terminal interaction that renders the central actin polymerization domain inactive. This autoinhibition is relieved upon the interaction with an active Rho GTPase. Sonja Kühn and Matthias Geyer ${ }^{14}$ discuss the current knowledge on the structure, function, and activation mechanism of formins as downstream effectors of Rho GTPases.

The $\mathbf{p} 21$ activated kinase (PAK) family of serine/threonine kinases are effector proteins for the Rho GTPases Cdc42 and Rac. The six mammalian PAKs can be categorized into two subgroups: group I (PAK 13) and group II (PAK 4-6), with structurally distinct regulatory domains. Some of the PAKs are ubiquitously expressed whereas some others have more restrictive tissue specificity, but all of them are present in the nervous system. These two subfamilies have both overlapping and distinct functions, and are regulated by different autoinhibitory mechanisms. Through their interactions with a wide array of intracellular proteins, PAKs are involved in multiple signaling pathways, particularly in cytoskeletal organization, cell migration, survival and mitosis. Chetan K Rane and Audrey Minden ${ }^{15}$ discuss the basic structures of the PAKs, and their roles in cell growth, development, and in cancer.

Phosphatidylinositol 3-kinases (PI3Ks) are lipid kinases that produce phosphorylated lipids which, in turn, transduce extracellular cues within the cell and are involved in many cellular functions such as cell growth, proliferation, differentiation, motility, survival and intracellular trafficking, which in turn are involved in cancer. Rac is a Rho GTPase that impacts on actin organization, but PI3K and Rac signaling pathways frequently intermingle. For instance, phosphorylated lipids produced by PI3Ks recruit and activate GEFs and GAPs, key modulators of Rac function whereas PI3Ks interact with activated Rac, leading to Rac signaling amplification. C.C. Campa, E. Ciraolo, A. Ghigo, 
G. Germena and E. Hirsch ${ }^{16}$ summarizes the molecular mechanisms underlying the cross-talk between Rac and PI3K signaling in two different processes, cell migration and reactive oxygen species (ROS) production.

Phagocytic cells (as neutrophils or macrophages) destroy engulfed pathogens by multiple effector mechanisms. An important component of them is the ability of phagocytic cells to generate ROS via a membrane-associated NADPH oxidase in response to receptor agonists such as growth factors or inflammatory cytokines that signal through the Rho-like small GTPases Rac1 or Rac2. NADPH oxidase is a multicomponent enzyme that utilizes electrons derived from intracellular $\mathrm{NADPH}$ to generate superoxide anion, which subsequently dismutes to $\mathrm{H}_{2} \mathrm{O}_{2}$ and results in the formation of other ROS that are used for host defense. In cell-free systems, either Rac1 or Rac2 GTPase is required for oxidase activity. Edgar Pick ${ }^{17}$ focus on the role the small GTPase Rac in oxidase activation in phagocytic cells.

\section{Cellular Signaling Networks and Rho GTPases: Major Crossroads}

Rho GTPases regulate a diverse range of cellular functions primarily through their ability to modulate microtubule dynamics and the actin-myosin cytoskeleton. Microtubules (MTs) are highly versatile, dynamic polymers mainly composed of $\alpha / \beta$ tubulin heterodimers, arranged head to tail to form $20 \mathrm{~nm}$ hollow cylindrical tubes. Virtually involved in every aspect of cell biology, MTs typically undergo cycles of rapid growth and disassembly, a phenomenon known as dynamic instability, in response to environmental cues. Rho GTPases play major roles in modulating MT stability, but MTs have also emerged as key regulators of RhoGTPase functioning. J. Wojnacki, G. Quassollo, M.P. Marzolo and A. Cáceres ${ }^{18}$ summarize the key studies describing the reciprocal relationship between small Rho-GTPases and MTs during migration and polarization.

Owing to their ability to control the assembly and organization of the actin and to modulate microtubule dynamics,
Rho GTPases are known to regulate mitosis and cytokinesis progression. Mitosis results in the generation of two independent daughter cells. After division of the cell nucleus into two identical sets of chromosomes, each in its own nucleus, the cytokinesis is the mechanical process which cleaves the mother cell into two daughter cells at the end of the cell cycle. Mitotic progression involves dynamic modulation of cell shape primarily through remodeling of the actin and microtubule cytoskeletons. Megan Chir$\operatorname{cop}^{19}$ reviews the molecular mechanisms regulating the spatial and temporal activation of RhoA and Cdc42 during mitosis, and how this is critical to control cell division progression.

Cell polarity refers to the asymmetric organization of different aspects of the cell, including the cell surface, the intracellular organelles and the cytoskeleton, which allow the cell to perform specialized cellular functions. Cell polarity is initiated by spatial cues, interpreted and relayed by signaling cascades to the actin cytoskeleton. Signaling via the Rho GTPases provides crucial regulation of numerous cell polarization events, including apicobasal polarity, polarized cell migration, polarized cell division and neuronal polarity. Natalie A. Mack and Marios Georgiou ${ }^{20}$ discuss the intimate relationship between the Rho GTPases and apicobasal cell polarity, highlight the interdependent nature of their regulation and draw upon evidence from a variety of systems and experimental approaches.

The ability of Rho GTPases to interact with membranes allows them to target actin polymerization to discrete sites on the plasma membrane and to intracellular membrane compartments and thereby to induce membrane protrusions and to regulate vesicular transport between cell compartments. Among their potential binding partners or effectors, phosphatidylinositol and its phosphorylated derivatives, phosphoinositides, are among the most versatile regulatory molecules in eukaryotic cells, playing key roles in the modulation of membrane trafficking, intracellular signaling and cytoskeleton organization. P. Croisé, C. Estay-Ahumada, S. Gasman and S. Ory ${ }^{21}$ highlight recent advances in our understanding of how Rho-dependent signaling pathways organize actin dynamics and phosphoinositides and how phosphoinositides potentially provide negative feedback to Rho GTPases during endocytosis, exocytosis and membrane exchange between intracellular compartments.

The remarkable ability of a single axon to extend multiple branches and form terminal arbors enables vertebrate neurons to integrate information from divergent regions of the nervous system. Branches emerge from cell bodies after an initial polarization stage, elongate, and navigate towards target regions guided by a range of environmental cues, a process culminating in synapse formation. While axonal branching is well described, the mechanisms that control it are still only partially understood. The cytoskeletal mechanisms of axonal branching are orchestrated by Rho GTPases. Mirela Spillane and Gianluca Gallo ${ }^{22}$ provide a survey of the roles of Rho-family GTPases in mechanisms of axon collateral branching by primary neurons.

Cell migration is an essential process in all multicellular organisms, important not only during development, but also throughout life such as in wound repair, during immune surveillance as well as during cancer progression. It involves rearrangements of cellular architecture via dynamic and spatially regulated changes to the cytoskeleton and cell adhesion proteins. Specific RhoGTPase signaling governs spatio-temporal activation and coordination of subsequent protein-protein and protein-lipid interactions. Amine Sadok and Chris J. Marshall ${ }^{23}$ give an overview of recent studies on the role of Rho-family GTPase members in the modulation of cell migration in different environments, and discuss future directions.

Phagocytosis is the process whereby cells engulf large particles to form internal vesicles termed phagosomes. The ultimate goal of anti-pathogen phagocytosis is to destroy engulfed bacteria or fungi and to stimulate cell-cell signaling that mount an efficient immune defense. Clearance phagocytosis of apoptotic cells and cell debris is important to inhibit inflammation and autoimmune responses against intracellular antigens. All forms of phagocytosis, regardless of type of particle and phagocytic cells, require $\mathrm{F}$-actin recruitment beneath tethered particles and 
F-actin re-arrangement promoting engulfment, which are controlled by Rho family GTPases. Yingyu Mao and Silvia C. Finnemann ${ }^{24}$ examine the specificity of Rho GTPase activity at the different steps of phagocytosis by mammalian cells.

Actin, one of the major components of the cytoskeleton is also, besides its mechanical functions, involved in the regulation of transcription and gene expression. Nuclear actin can indeed regulate the access of the transcription machinery to the genes. The nuclear and cytoplasmic actin pools now appears to be in dynamic communication. As actin and actin binding proteins, that regulate different aspects of actin polymerization (e.g. filament nucleation, severing and depolymerization) are major downstream targets of Rho GTPases, the latter may therefore regulate gene expression by controlling either cytoplasmic or nuclear actin dynamics. Eeva Kaisa Rajakylä and Maria K. Vartiainen ${ }^{25}$ discuss the possible mechanisms and key proteins that may mediate the transcriptional regulation by Rho GTPases through actin.

Effective immune response against pathogenic agents requires rapid and specific threat detection and intercellular communication. In many cases, immune cells accomplish these goals by building specialized, transient interactions with each other and also with other cells in the surrounding tissue. This often requires them to completely reorganize their cellular architecture. $\mathrm{T}$ cells (lymphocytes which mature in the thymus), major actors in the immune system, exemplify this structural plasticity by adopting drastically different structural configurations. Rho GTPases represent key regulators of T lymphocyte biology. A. Saoudi, S. Kassem, A.S. Dejean and G. Gaud ${ }^{26}$ highlight the roles of Rho-GTPases in the thymic development of $\mathrm{T}$ cells, in $\mathrm{T}$ cell migration in lymphoid organs or inflammatory tissues and in $\mathrm{T}$ cell activation and differentiation.

\section{Tissue Signaling Networks Involving Rho GTPases}

Neural crest cells are a multipotent migratory stem cell population unique to vertebrates, which gives rise to most of the peripheral nervous system and to several non-neural cell types (e.g. smooth muscle cells of the cardiovascular system, etc.). These cells originate in the ectoderm at the border of the neural tube, undergo an epithelial-mesenchymal transition and acquire outstanding individual and collective migratory properties that allow them to disseminate and differentiate to different parts of the body. Philippe Fort and Eric Théveneau ${ }^{27}$ show how signaling pathways controlled by Rho GTPases, key players in cell adhesion, contraction, migration and polarity, contribute to the control of the different phases of neural crest induction, delamination and migration.

Bone is a dynamic tissue subjected to a continuous renewing through a regulated balance between activities of osteoclasts, which resorb mineralized bone, and osteoblasts, which form the new bone matrix. Osteoclasts are large multinucleated cells derived from hematopoietic stem cells via a complex differentiation process. The osteoclast cytoskeleton is a unique structure that polarizes the cell's resorptive machinery to the bone-cell interface where it creates an isolated resorptive microenvironment consisting of an actin ring surrounding a ruffled border. Protons and proteases secreted within this microenvironment demineralize and degrade bone. Heiani Touaitahuata, Anne Blangy and Virginie Vives ${ }^{28}$ highlight the roles of Rho GTPases in all the steps of osteoclast differentiation, function, and death.

Epithelial cells are a specialized cell type that form selectively permeable barriers between different compartments of a multicellular organism. The apical junctional complex, encompassing the tight junctions and adherens junctions, plays a vital role in regulating epithelial cell differentiation and barrier function of epithelial organs. Apical junctions are supported, shaped and regulated by cytoskeletal filaments, whose dynamic organization and contractility are finely tuned by GTPases of the Rho family. S. Citi, D. Guerrera, D. Spadaro and J. Shah ${ }^{29}$ propose the concept of "zonular signalosome" to highlight the close functional relationship between proteins of apical epithelial junctions and the control of cytoskeletal organization and signaling through Rho GTPases, transcription factors, and their effectors.

Cell adhesion to the surrounding microenvironment is crucial for force transfer to and from the cell. Each of the two interfaces of cellular adhesion, cellcell and cell-extracellular matrix (ECM) adhesion, has unique components but both link directly to the cell's actin cytoskeleton. Integrins sense the physical properties of the extracellular matrix and organize the cytoskeleton accordingly but also regulate the activity of several Rho GTPases and of members of the Src family of tyrosine kinases. Campbell D. Lawson and Keith Burridge ${ }^{30}$ present the recent advances in our understanding of the role of mechanical tension in the development and maturation of cell adhesion and the crosstalk that exists between integrins and Rho GTPases in mediating these force-dependent responses.

Insulin, secreted into blood vessels from $\beta$ cells of pancreatic islets in response to high blood glucose levels, stimulates an array of physiological responses in target tissues, including liver, skeletal muscle, and adipose tissue, thereby reducing the blood glucose level. Insulin-dependent glucose uptake in skeletal muscle and adipose tissue is primarily mediated by the redistribution of the glucose transporter type 4 (GLUT4), which cycles between the intracellular compartments and the plasma membrane. The cell cytoskeleton has substantial influence over vesicle trafficking events, and different Rho (e.g. Rac1, TC10) and Rab GTPases have been found to be insulin-activated and/or have been assigned various functions in insulinstimulated GLUT4 translocation. Takaya Satoh $^{31}$ examines the involvement of Rho GTPases in insulin-stimulated glucose uptake.

The ability to reorganize their cytoskeleton is a fundamental property of embryonic cells. Their shape changes during their development (during gastrulation and dorsal closure for example) indeed depend on the plasticity of the actin cytoskeleton, and the ability of cells or cell extensions (e.g. axons) to migrate within the developing embryo requires rapid and spatially organized changes to the actin cytoskeleton in response to the external 
environment. Rho GTPases play key roles in signal transduction pathways that link plasma membrane receptors to the organization of the actin cytoskeleton. Philippe M. Duquette and Nathalie Lamarche$V_{a n e}{ }^{32}$ summarize the recent findings regarding Rho functions in embryonic development, with emphasis on early embryogenesis, epithelial and skin morphogenesis, tubulogenesis, development of the central nervous system, and limb development.

\section{Rho GTPase Signaling in the Development and Progression of Diseases}

Essential hypertension, a major risk factor and one of the most common cardiovascular diseases, is characterized by significant and persistent elevations in arterial pressure. Hypertension is a multifactorial disorder that may involve abnormalities in the functions of the heart pump, the blood vessels, and the kidneys. Members of the Rho family of small GTPases (in particular RhoA and Rac1) are recognized key signaling molecules playing important roles in the highly coordinated regulation processes controlling arterial blood pressure. Gervaise Loirand and Pierre Pacaud ${ }^{33}$ provide an overview of the current state of knowledge on the involvement of Rho GTPase signaling in the control of blood pressure and the pathogenesis of hypertension.

Tumor progression involves the transition from normal to malignant cells by a series of cumulative alterations resulting in the acquisition of six biological capabilities. These hallmarks consist of sustaining proliferative signaling, evading growth suppressors, resisting cell death, enabling replicative immortality, inducing angiogenesis, and activating invasion and metastasis programs. Key regulator of the actin cytoskeleton, the Rho family plays fundamental roles since alterations to the actin cytoskeleton cause changes in cell adhesion, migration, and invasion, resulting in epithelial-mesenchymal or mesenchymal-epithelial transition, metastasis, neoangiogenesis, and infiltration of immune cells. Jose L. Orgaz, Cecilia Herraiz and Victoria Sanz-Moreno ${ }^{34}$ focus their attention on the contribution of Rho GTPases to cell cycle progression, oncogenic transformation, tumor suppression, cancer cell survival, senescence, inflammation, angiogenesis, and altered metabolism.

Cell migration is a fundamental process controlling cell position, fate decisions, and function in morphogenesis, immune function, regeneration, and cancer. Podosomes and invadopodia (which can be subsumed under the common term "invadosomes") are specialized plasmamembrane actin-based microdomains that establish close contact with the ECM but are also, in contrast to similar structures (e.g. focal adhesions), able to degrade components of this ECM. Invadosomes are, therefore, considered as key structures of cell invasion. Adhesive and soluble ligands act via transmembrane receptors that propagate signals to the cytoskeleton via small $G$ proteins of the Rho family, assisted by tyrosine kinases and scaffold proteins to induce invadosome formation and rearrangements. P. Spuul, P. Ciufici, V. Veillat, A. Leclercq, T. Daubon, I. Kramer and E. Génot ${ }^{35}$ provide an overview of the importance of Rho GTPases in formation, characteristics, and functions of invadosomes. Some cells migrate as individuals, but many cell types can migrate collectively in tightly or loosely associated groups. Collective migration serves to keep the tissue intact during remodeling, allows mobile cells to carry other cells that are otherwise immobile, and ensures appropriate distribution of cells within a tissue. A cell, initially similar to the others, becomes a leader cell while its neighbors do not alter. The determinants of these leader cells include mechanical and biochemical cues, often under the control of small GTPases. Mirjam M. Zegers and Peter Friedl ${ }^{36}$ summarize key functions of Rho GTPases in collective cell migration, with focus on their contribution to leader cell polarity, cell-cell junction stability and turnover, and multicellular coordination during morphogenesis and cancer.

Tumors are heterogeneous cellular entities composed of cancer cells and of cells of the microenvironment in which they reside. A reciprocal dynamic interaction occurs between the tumor cells and their surrounding stroma to promote cancer development, metastasis and chemoresistance. The role of this complex tumor microenvironment in driving the aggressiveness of the tumor is increasingly being realized. Given their importance in the regulation of cell shape, cytokinesis, cell adhesion, and cell motility, Rho GTPases may then play key roles at the tumor-stromal interface in disease progression and therapeutic targeting. M. Pajic, D. Herrmann, C. Vennin, J.R.W. Conway, V.T. Chin, A.K.E. Johnsson, H.C.E. Welch and P. Timpson ${ }^{37}$ explain how to target Rho signaling might provide interesting avenues for the development of combination therapies.

\section{Rho GTPase as Pathogen Targets; Therapeutic Perspectives}

The cytoskeleton of eukaryotic cells plays crucial roles in host-pathogen interactions. It is essential for epithelial barrier functions, phagocytosis and regulation of immune cell signaling. The cytoskeleton of eukaryotic cells has a pivotal role in host-pathogen interactions. Among the multiple regulation steps of the actin cytoskeleton, bacterial factors interact preferentially with Rho GTPases. Pathogens either release toxins in the surrounding environment or directly inject virulence factors into target cells. Bacterial toxins interfering with Rho GTPases, and to some extent with Ras GTPases catalyze a covalent modification (ADPribosylation, glucosylation, deamidation, adenylation, proteolysis) which block them in their active or inactive state, resulting in alteration of epithelial and/or endothelial barriers, which contributes to dissemination of bacteria in the host. Michel R. Popoff ${ }^{38}$ examines the diversity of RhoGTPase manipulations by bacterial pathogens to disseminate and survive in host. Several microorganisms modulate the eukaryotic cell surface to facilitate their engulfment. Once internalized, they hijack the molecular machinery of the infected cell not only to prevent their own degradation in the phagocytic pathway, but also to create a favorable intracellular environment for growth and replication. Cristián Quintero, Julián Gambarte, María Damiani ${ }^{39}$ 
focus their attention on the role of Rho proteins on four curable, sexually transmitted infections, Chlamydia trachomatis, Neisseria gonorrhoeae, Trichomonas vaginalis and Treponema pallidum.

The small size, structural simplicity, and lack of any metabolic or motile activities of viruses severely limit the types of processes that they can themselves undertake to promote the transfer process from infected to uninfected cells and from

\section{References}

1. Madaule P, Axel R. A novel ras-related gene family. Cell. 1985; 41:31-40.

2. Rubin EJ, Gill DM, Boquet P, Popoff MR. Functional modification of a 21-kilodalton G protein when ADPribosylated by exoenzyme C3 of Clostridium botulinum. Mol Cell Biol. 1988; 8:418-26.

3. Chardin P, Boquet P, Madaule P, Popoff MR, Rubin EJ, Gill DM. The mammalian G protein rhoC is ADPribosylated by Clostridium botulinum exoenzyme C3 and affects actin microfilaments in Vero cells. EMBO J. 1989; 8:1087-92.

4. Ridley AJ, Hall A. The small GTP-binding protein rho regulates the assembly of focal adhesions and actin stress fibers in response to growth factors. Cell. 1992;70:389-99.

5. Ridley AJ, Paterson HF, Johnston CL, Diekmann D, Hall A. The small GTP-binding protein rac regulates growth factor-induced membrane ruffling. Cell. 1992;70:401-10.

6. Schmidt S, Debant A. Function and regulation of the Rho guanine nucleotide exchange factor Trio. Small GTPases. 2014; 5: e29769.

7. Bustelo XR. Vav family exchange factors: an integrated regulatory and functional view. Small GTPases. 2014; 5:9.

8. Welch HCE. Regulation and Function of P-Rex Family Rac-GEFs. Small GTPases. In press.

9. Hobbs GA, Zhou B, Cox AD, Campbell SL. Rho GTPases, oxidation, and cell redox control. Small GTPases. 2014;5:e28579.

10. Aspenström P. BAR domain proteins regulate Rho GTPase signaling. Small GTPases. 2014; 5: 7.

11. Schaefer A, Reinhard NR, Hordijk PL. Toward understanding RhoGTPase specificity: structure, function and local activation. Small GTPases. 2014; 5: 6.

12. Julian L, Olson MF. Rho-associated coiled-coil containing kinases (ROCK): structure, regulation, and functions. Small GTPases. 2014; 5: e29846.

13. Zhao ZS, Manser E. Myotonic dystrophy kinaserelated Cdc42-binding kinases (MRCK), the ROCKlike effectors of Cdc42 and Rac1. Small GTPases. In press. infected to noninfected host organisms. A key step of the conversion of cell normal functions to optimize viral replication and virion production is the reconfiguration and reorganization of cellular actin, affecting every stage of the viral life cycle, from entry through assembly to egress. Given the diversity between viruses, there is considerable variability in viral interactions with Rho GTPase signaling, the major regulator of actin and microtubule

14. Kühn S, Geyer M. Formins as effector proteins of Rho GTPases. Small GTPases. 2014; 5: e29513.

15. Rane CK, Minden A. P21 activated kinases: structure, regulation, and functions. Small GTPases. 2014; 5. pii: e28003.

16. Campa CC, Ciraolo E, Ghigo A, Germena G, Hirsch E. Crossroads of PI3K and Rac pathways. Small GTPases. In press.

17. Pick E. Role of the Rho GTPase Rac in the activation of the phagocyte NADPH oxidase: outsourcing a key task. Small GTPases. 2014; 5: e27952.

18. Wojnacki J, Quassollo G, Marzolo MP, Cáceres A. Rho GTPases at the crossroad of signaling networks in mammals: impact of Rho-GTPases on microtubule organization and dynamics. Small GTPases. 2014; 5: e28430.

19. Chircop M. Rho GTPases as regulators of mitosis and cytokinesis in mammalian cells. Small GTPases. 2014; 5. pii: e29770

20. Mack NA, Georgiou M. The interdependence of the Rho GTPases and apicobasal cell polarity. Small GTPases. 2014; 5: 10.

21. Croisé P, Estay-Ahumada C, Gasman S, Ory S. Rho GTPases, phosphoinositides, and actin: a tripartite framework for efficient vesicular trafficking. Small GTPases. 2014; 5: e29469.

22. Spillane M, Gallo G. Involvement of Rho-family GTPases in axon branching. Small GTPases. 2014; 5: e27974.

23. Sadok A, Marshall CJ. Rho GTPases: masters of cell migration. Small GTPases. 2014;5: e29710.

24. Mao Y, Finnemann S. Regulation of phagocytosis by Rho GTPases. Small GTPases. In press.

25. Rajakylä EK, Vartiainen MK. Rho, nuclear actin, and actin-binding proteins in the regulation of transcription and gene expression. Small GTPases. 2014; 5: e27539.

26. Saoudi A, Kassem S, Dejean A, Gaud G. Rho-GTPases as key regulators of $\mathrm{T}$ lymphocyte biology. Small GTPases. 2014;5. pii: e28208.

27. Fort P, Théveneau E. PleiotRHOpic: Rho pathways are essential for all stages of Neural Crest development. Small GTPases. 2014; 5: e27975. dynamics and organization. Céline Van den Broeke, Thary Jacob and Herman W. Favoreel $^{40}$ review the known mechanisms utilized by viral pathogens to manipulate Rho GTPase signaling in order to facilitate their entry, replication, and spread.

We wish to thank all authors and co-authors for their commitment and the anonymous reviewers who contributed by their critical constructive remarks to the excellence of this issue.

28. Touaitahuata H, Blangy A, Vives V. Modulation of osteoclast differentiation and bone resorption by Rho GTPases. Small GTPases. 2014; 5: e28119.

29. Citi S, Guerrera D, Spadaro D, Shah J. Epithelial junctions and Rho family GTPases: the zonular signalosome. Small GTPases. 2014; 5: 1-15.

30. Lawson CD, Burridge K. The on-off relationship of Rho and Rac during integrin-mediated adhesion and cell migration. Small GTPases. 2014; 5: e27958.

31. Satoh T. Rho GTPases in insulin-stimulated glucose uptake. Small GTPases. 2014; 5: e28102.

32. Duquette PM, Lamarche-Vane N. Rho GTPases in embryonic development. Small GTPases. 2014; 5: 8.

33. Loirand G, Pacaud P. Involvement of Rho GTPases and their regulators in the pathogenesis of hypertension. Small GTPases. 2014: e28846.

34. Orgaz JL, Herraiz C, Sanz-Moreno V. Rho GTPases modulate malignant transformation of tumor cells. Small GTPases. 2014; 5: e29019.

35. Spuul P, Ciufici P, Veillat V, Leclercq A, Daubon T, Kramer I, Génot E. Importance of RhoGTPases in formation, characteristics, and functions of invadosomes. Small GTPases. 2014; 5: e28195.

36. Zegers MM, Friedl P. Rho GTPases in collective cell migration. Small GTPases. 2014; 5: e28997.

37. Pajic M, Herrmann D, Vennin C, Conway JRW, Chin VT, Johnsson AKE, Welch HCE, Timpson P. The dynamics of Rho GTPase signalling and implications for targeting cancer and the tumour microenvironment. Small GTPases. In press.

38. Popoff MR. Bacterial factors exploit eukaryotic Rho GTPase signaling cascades to promote invasion and proliferation within their host. Small GTPases. 2014; 5. pii: e2820.

39. Quintero C, Gambarte J, Damiani M. Rho GTPases as pathogen targets: focus on curable sexually transmitted infections. Small GTPases. In press.

40. Van den Broeke C, Jacob T, Favoreel HW. Rho'ing in and out of cells: viral interactions with Rho GTPase signaling. Small GTPases. 2014; 5: e28318. 